Jan Stenger

\title{
Sitzen bleiben oder aufstehen? Caesars symbolische Kommunikation auf der Bühne des Forum Iulium (Suet. Iul. 78)
}

\section{Symbolische Körperpraktiken in der Moderne und in Rom}

Nachdem im türkischen Fernsehen eine gewalttätige Serie mit eindeutig anti-israelischer Tendenz ausgestrahlt worden war, kam es Anfang des Jahres 2010 zu einem Eklat zwischen den offiziell miteinander befreundeten Ländern Israel und Türkei. Um sich gegen die Hetze zur Wehr zu setzen, bestellte der israelische Außenminister Lieberman den türkischen Botschafter Ahmet Çelikkol in die Knesset ein. Dort ließ man ihn einige Zeit vor der Tür warten, bis ihn der stellvertretende Außenminister Danny Ayalon mit zwei Mitarbeitern empfing. Zu seiner Überraschung wurde der Botschafter auf einem äußerst niedrigen Sofa plaziert, in dem er förmlich versank, während Ayalon und seine Mitarbeiter in aufrechter Sitzposition auf höheren Stühlen Platz nahmen (Abb. I). Um die Symbolik der Szene vollends augenfällig zu machen, hatte man auf dem Tisch ausschließlich die israelische Flagge aufgestellt. ${ }^{\mathrm{T}}$

Während man im Allgemeinen gerade im Bereich der Diplomatie darauf Wert legt, gewisse Regeln der Kooperation zu beachten, verstieß Israel gegen die Gepflogenheiten, und zwar gezielt, um die Türkei für die Provokation der Fernsehserie zu bestrafen. Statt sich auf verbale Kritik am türkischen Fernsehen zu beschränken und eine Änderung zu verlangen, setzte das israelische Außenministerium auf eine symbolische Handlung, welche die gewünschte Hierarchie zwischen beiden Staaten visualisieren sollte. Die nonverbale Kommunikation diente in erster Linie nicht der Informationsübermittlung, sondern transportierte als eigentliche Botschaft die Demütigung des Kontrahenten und brachte eine asymmetrische Beziehung zum Ausdruck.

In anderen Kontexten würde man solche ungleichen Sitzverhältnisse vielleicht als Ungeschicklichkeit oder Fauxpas erachten, nicht jedoch im zwischenstaatlichen Verkehr. Dass die Plazierung des Botschafters alles andere als zufällig war, erhellt zudem aus der Anwesenheit der Medien. Von den Usancen abweichend, hatte man zu dieser Einbestellung eines Botschafters das Fernsehen hinzugebeten, damit die Demütigung von der gesamten israelischen Öffentlichkeit und möglichst auch von der Weltöffentlichkeit wahrgenommen wurde. Und der Vizeaußenminister erklärte den Medienvertretern unverhohlen: „Haupt-

I Siehe den Bericht von Clemens Verenkotte, „Israel brüskiert gezielt türkischen Botschafter“ http://www. tagesschau.de/ausland/israel792.html (Stand I6. o8. 20I0); ferner http://www.heute.de/ZDFheute/inhalt/ 30/0,3672,8010718,00.html (Stand I5. 02. 2010). 


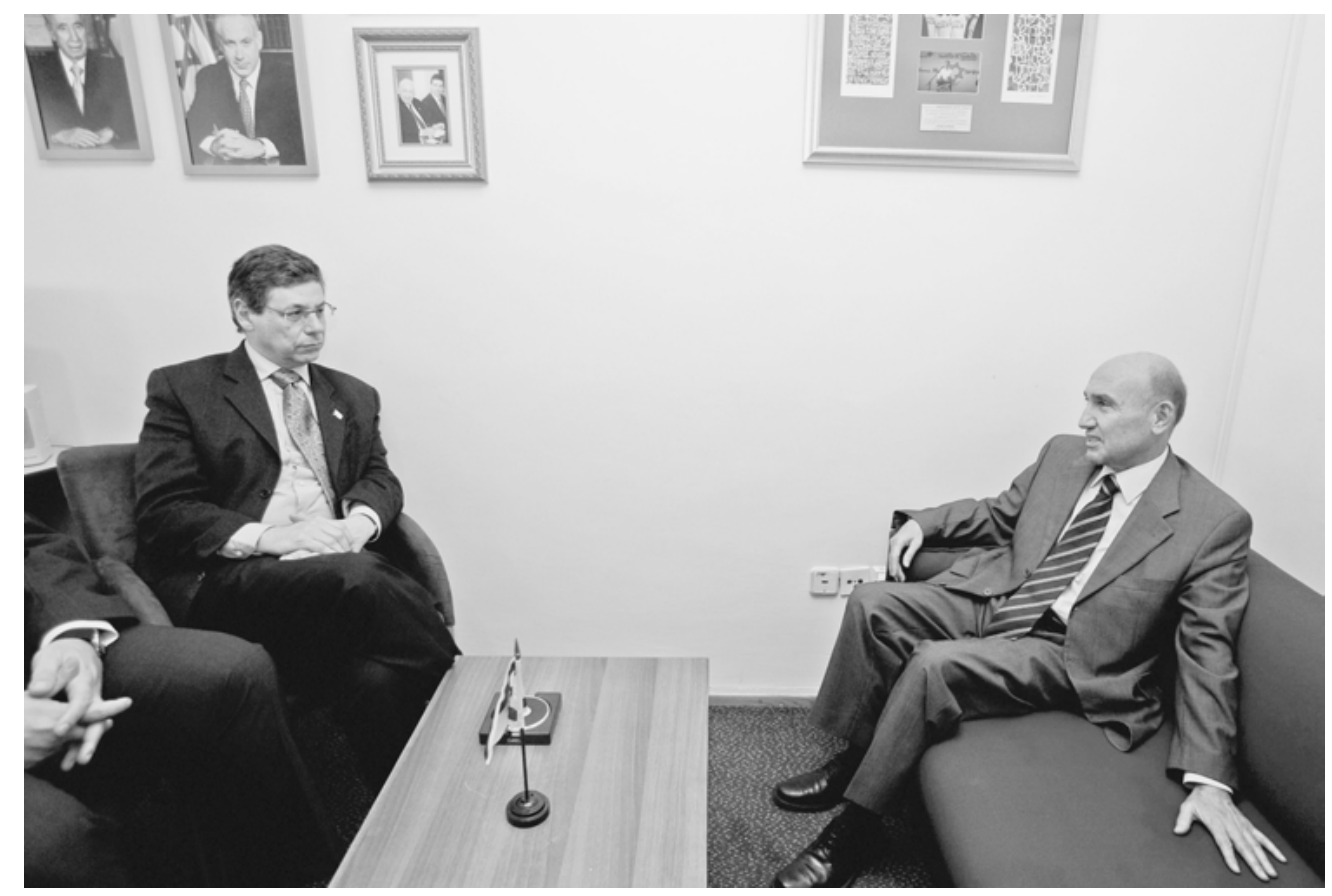

Abb. 1 | Daniel Ayalon, stellv. Außenminister Israels und Ahmet Çelikkol, Botschafter der Türkei

sache man sieht, dass er tief sitzt und wir hoch und dass nur eine Flagge da ist. Und ihr seht, dass wir nicht lächeln.“2 Man kalkulierte von vornherein ein großes Publikum ein, ohne das die symbolische Kommunikation nur begrenzt funktioniert hätte. Wäre keine Fernsehkamera zugegen gewesen, hätte die Kunde von der Demütigung möglicherweise niemals das Büro des Außenministers verlassen.

Wie gewünscht erntete die Begebenheit einige Aufmerksamkeit, und es entspann sich eine rege Diskussion über den ungewöhnlichen Umgang mit dem Repräsentanten eines Staates. Wer auf einem diffizilen Terrain wie dem diplomatischen Parkett geschriebene oder ungeschriebene Regeln bricht, so lehrt die Episode, kann damit rechnen, genauestens beobachtet und von seinen Beobachtern bewertet zu werden, sei es positiv im Sinne von Anerkennung und Zustimmung, sei es negativ durch Kritik und Ablehnung. Nicht anders erging es dem amerikanischen Präsidenten Barack Obama, als er sich in demonstrativ legerer Pose auf einer Treppe liegend im Kreise seiner Berater ablichten ließ und dafür in der Öffentlichkeit seines Landes scharf gerügt, aber auch gefeiert wurde. 3 Gemeinsam ist beiden Vorkommnissen, dass sie sich in einem Kontext ereigneten, in dem mit Argusaugen

2 http://www.tagesschau.de/ausland/israel792.html.

3 Vgl. Peter Richter, „Obama sitzt. Ikonographie eines Präsidenten“, Frankfurter Allgemeine Sonntagszeitung, 06. 09. 2009 (auch http://www.faz.net/-oomjf4 [Stand 6. I2. 20I0]). 
auf Hierarchien, Umgangsformen und Symbolik geachtet wird. Vertreter eines Staates agieren in der Öffentlichkeit niemals als reine Privatpersonen, sondern spielen eine Rolle gemäß einem fein ausgeklügelten Drehbuch. Bestandteile der inszenierten Szenen sind fast immer Zeremonien und Rituale, denen große Symbolkraft innewohnt, die mehr bedeuten, als sie an der Oberfläche zu erkennen geben. Zudem sind solche Momente, eben weil sie Normen und Regeln folgen, besonders heikel und für Störungen anfällig, wenn einer der Akteure aus der Rolle fällt und den Erwartungen des Publikums nicht gerecht wird. Die sorgfältig austarierte Balance wird erschüttert und droht die Akteure oder zumindest einige der Beteiligten zu beschädigen.

Wenn hier soeben Begriffe wie Akteure, Rollen, Szenen und Inszenierung der Sphäre des Theaters entlehnt worden sind, so sind sie nicht ohne Bedacht als alltägliche Metaphorik gewählt worden, sondern sollen darauf aufmerksam machen, dass menschlichen Interaktionen, zumal solchen, die in hohem Maße formalisiert sind, stets ein theaterhafter Zug eignet. Begegnen sich Menschen in verschiedenen Kontexten, so verkörpern sie bestimmte Rollen, nach denen sie ihr Auftreten und ihre Verhaltensweisen und Handlungen gestalten. Sofern sie ihre Rolle mit Bravour ausfüllen, bestätigen sie die Erwartungen ihrer Mitspieler und des Publikums, so dass das Stück störungsfrei über die Bühne geht.4 Da in sozialen Interaktionen Machtverhältnisse oder gesellschaftliche Relationen ausgehandelt und durch ritualisierte Handlungen reproduziert werden, spielen die Beteiligten nachgerade automatisch Theater und gehorchen Rollenmustern. Ebenso ist es indessen denkbar, durch gezielte Verletzung von Normen Machtverhältnisse zu den eigenen Gunsten zu verändern, indem man seine Mitspieler düpiert. Wie das von vielen als unziemlich empfundene Gebaren des amerikanischen Präsidenten demonstriert, kann mitunter das intendierte und nicht einmal präsente Publikum als eigentlicher Adressat der Handlung zum Mitspieler avancieren, da den anwesenden Beratern lediglich die Rolle von Statisten zugedacht war. Im Zuge des von ethnologischer Ritualanalyse und Theaterwissenschaft angestoßenen performative turn hat die kulturwissenschaftliche Forschung mit den Kategorien der Theatralität und der Performanz unseren Blick für solche sozialen Phänomene geschärft und das Feld auch für sozialwissenschaftlich beeinflusste Interpretationen von Texten bereitet.5 Die aus dem Theater geborgten Konzepte erlauben es, vielfältige soziale Begegnungen adäquat zu analysieren und die zugrunde liegenden Kommunikationsakte zu verstehen.

Symbolträchtige Handlungen, insbesondere Regelverstöße, sind nicht allein in der heutigen Diplomatie und Politik auf Schritt und Tritt anzutreffen, sondern kennzeichnen ebenso die politischen Verhältnisse im republikanischen und kaiserzeitlichen Rom. Die antike Historiographie ist voll von Begebenheiten, in denen römische Politiker im öffentlichen Raum in Tränen ausbrechen, ihre Brust entblößen und andere Handlungen mit ih-

4 Rollenmuster, Normen und Rituale entlasten also auch die Kommunikation, indem sie als eine Art Orientierungswissen durch ihre strukturierende Wirkung deren Komplexität reduzieren.

5 Goffman (I969); Goffman (I986); Turner (I989); Fischer-Lichte (2004); Bachmann-Medick (2006) I04-I43. 
rem Körper ausführen, die eine latente Botschaft transportieren und bestimmte politische Ziele durchsetzen sollen. ${ }^{6}$ Sei es, dass der Feldherr Pompeius vor seinen Truppen Tränen vergießt,7 sei es, dass Kaiser Titus durch Gesten mit der Volksmenge Scherze austauscht, ${ }^{8}$ so werden wir Zeugen, wie die verbale Kommunikation immer wieder durch Mimik, Gestik und Körpersprache ergänzt oder gar ersetzt wird. In der althistorischen Forschung der letzten Jahre haben performative Akte einige Aufmerksamkeit auf sich gezogen, wobei sich Vertreter einer solchen semiotischen bzw. ,praxeologischen Geschichtsschreibung' wie Egon Flaig an vorwiegend französische Ansätze der Anthropologie und der Soziologie anlehnen. ${ }^{9}$ Stärker als zuvor ist durch diesen Ansatz bewusst geworden, dass in einer nur teilweise alphabetisierten bzw. schriftlichen Kultur wie der römischen, in der politische Kommunikation weitgehend auf dem direkten Kontakt der Beteiligten basierte, die nonverbale Kommunikation durch Körperpraktiken ein kaum zu überschätzender Faktor politischer Beeinflussung ist. Eine eindrückliche Geste vermag unter Umständen mehr zu sagen, lauter zu sprechen als viele Worte.

Gleichwohl erhebt sich die Frage, ob es legitim ist, Konzepte wie Theatralität und Performanz oder erst recht die Kategorien des Normenverstoßes oder des Fauxpas auf fremde, vormoderne Kulturen zu übertragen, obwohl sie unverkennbar durch die Perspektive des Westens unserer Zeit geformt sind. Auch wenn kulturelle Differenzen zwischen unserer Gegenwart und dem Rom der Zeitenwende nicht geleugnet werden können, lässt sich dieser Ansatz auch für antike Phänomene fruchtbar machen, sofern man sich ins Gedächtnis ruft, dass die Verletzung einer sozialen Norm in der stark hierarchisierten römischen Gesellschaft ${ }^{\text {to }}$ mit ihren eigenen medialen Bedingungen und anderen Konzepten von Öffentlichkeit ${ }^{\text {II }}$ eine wesentlich andere Relevanz haben konnte als in der pluralistischen westlichen Kultur unserer Tage. ${ }^{12}$

6 Aus der immensen Zahl an einschlägigen Stellen seien hier nur genannt: Liv. 3,58,5f.; 5,30,4f.; 5,40,3; 45,20,6-9; Tac. ann. I,7,I; I,II,3; 4,8,2; 4,9,I; hist. I,82,I; 3,38,3; 3,58,3. Siehe Flaig (2003), bes. 99-136. Zu Ritualen und Verhaltensweisen in der Interaktion zwischen Ober- und Unterschicht in Rom siehe ferner Jehne (2000).

7 Plu. Pomp. $13,2$.

8 Suet. Tit. 8. Zur Gestik römischer Kaiser Aldrete (1999) 89-92.

9 Flaig (2003). Zu Gesten Aldrete (I999); Corbeill (2004); zu Tränen Fögen (2009); zur Körpersprache Cairns (2005). Siehe auch Bremmer u. Roodenburg (I992) und zur griechischen Literatur Boegehold (I999). Immer noch brauchbar die Materialsammlung von Sittl (I890).

Io Die hierarchische Ordnung der Gesellschaft zeigt sich insbesondere in dem streng reglementierten Gebrauch von Statussymbolen in Rom. Kolb (1977).

II Wenn in diesem Aufsatz die Begriffe ,öffentlich` bzw. ,Öffentlichkeit‘ gebraucht werden, geschieht dies in dem Bewusstsein, dass die Römer kein dem modernen Konzept der Öffentlichkeit, wie es etwa von Jürgen Habermas vertreten wird, vergleichbares Konzept besaßen. Als Öffentlichkeit wird hier der Bereich bezeichnet, in dem Einzelpersonen, Gruppen und Institutionen miteinander über Themen kommunizieren, die allgemein oder einem größeren Personenkreis zugänglich sind und von der Mehrheit der an der Diskussion beteiligten für relevant gehalten werden. Zur Diskussion über die Angemessenheit des Öffentlichkeitsbegriffs für die römische Gesellschaft Eich (2000).

I2 Insbesondere die römische Kaiserzeit und speziell das Agieren der Kaiser selbst sind unter dem Gesichtspunkt der Theatralität betrachtet worden. Siehe Bartsch (I994); Dupont (2003); Stenger (2010). 
Wie vertraut den Römern selbst es war, die öffentliche Verwendung der Körpersprache mit der Brille des Theaterbesuchers zu betrachten, kann der Blick auf die Rhetorik zeigen. Nicht nur bedienten sie sich des Begriffes persona, um in übertragenem Sinne soziale Rollen zu beschreiben, sondern sie nahmen auch die Nähe der Redner und politischen Akteure zu Schauspielern wahr. ${ }^{13}$ Wenn man durch Gesten oder im umfassenderen Sinne durch Körpersprache kommunizieren will, setzt dies voraus, dass es Rezipienten gibt, die kompetent sind, diese Zeichen zu lesen. Selbstverständlich existieren habituelle Praktiken des Körpers, die ohne weiteres innerhalb einer bestimmten Kultur verstanden werden, etwa das Weinen oder das Lachen. Darüber hinaus jedoch bedarf es eines feinen Gespürs für die zeichenhafte Bedeutung von Gesten, und zwar sowohl bei demjenigen, der sie einsetzt, als auch bei den intendierten Zuschauern. Während man heute in der politischen Beredsamkeit dem Körper nur wenig Beachtung schenkt und sich stattdessen auf das Wort konzentriert, steht es für die antike Rhetorik außer Frage, dass in der actio der Mimik, der Körperhaltung und der Gestik ein wichtiger Platz zufällt.. ${ }^{14}$ Da es sich bei der Rhetorik um eine systematische Kommunikationstheorie handelt, ist es unerlässlich, auch die nonverbalen Aspekte zu berücksichtigen, mit denen die Worte unterstützt, bisweilen sogar ersetzt werden können. Spricht schon Cicero in diesem Zusammenhang von sermo corporis und eloquentia corporis, so bietet Quintilian im elften Buch seiner Institutio oratoria eine ausgefeilte Theorie der Gestikulation des Redners. ${ }^{15}$ Auch wenn diese im Wesentlichen als Verstärkung der verbalen Kommunikation gedacht ist, zeigt sich Quintilian bewusst, dass die Gestik an die Stelle des Wortes treten könne und Praktiken des Körpers eigene Botschaften vermittelten, nicht zuletzt Respekt oder Missachtung gegenüber dem Publikum. ${ }^{16}$ Wenn die Rhetorik häufig darauf beharrt, die Grenze zur Schauspielkunst nicht zu überschreiten, dann offenbart sich darin doch unmissverständlich das Bewusstsein, wie eng rostra und scaena, Rednertribüne und Theaterbühne, miteinander verwandt sind. ${ }^{17}$ Ebenso wie die rhetorische Theorie geben auch Beschreibungen von Kommunikationssituationen oder bildliche Darstellungen einen plastischen Eindruck davon, wie selbstverständlich es für einen römischen Hörer war, Reden und Ansprachen sowohl mit dem Gehörsinn als auch mit seinen Augen zu folgen und den Auftritt des Redners in seiner Gesamtheit zu rezipieren. ${ }^{18}$ Dies gilt gleichermaßen für die Angehörigen der gebildeten Elite, die den entsprechenden Rhetorikunterricht genossen hatten, wie für die der einfachen Bevölkerung, der sich in der Stadt zahllose Gelegenheiten boten, Auftritten professioneller Redner beizuwohnen.

I3 Cic. off. I,I05-I25. Zum Begriff der persona Fuhrmann (I979); Gill (I988).

I4 Maier-Eichhorn (I989); Graf (I992); Wülfing (I995); Aldrete (I999) 3-84.

I5 Cic. de orat. 3,222; orat. 55; Quint. inst. II,3,65-I84.

I6 Quint. inst. II,3,85; I36.

I7 Zur Distanzierung der Rhetorik von der Bühnenkunst Quint. inst. II,3,89-9I; I03; I82-I84. Graf (I992) 39, 48-51.

I8 $\mathrm{Zu}$ den bildlichen Darstellungen Aldrete (I999) 45-50. 
Man könnte demnach mit Fug und Recht sagen, dass für die Körpersprache in der römischen Kultur eine entwickelte Grammatik existierte, die bei der Lektüre solcher performativen Akte half, damit man hinter der Körpersprache die Geisteshaltung des Gegenübers erkannte. ${ }^{19}$ Nicht vergessen werden darf, dass die Gestik der Redner genauso streng formalisiert war wie die der Schauspieler. Das Publikum war folglich mit einer sorgfältigen Inszenierung konfrontiert, nicht mit einer Improvisation. Zu Missverständnissen, Fehlschlägen und konträren Bewertungen kam es dennoch immer wieder. Das belegen nicht zuletzt die Beobachtungen der Geschichtsschreiber.

\title{
2. Caesars Sitzenbleiben als Normentransgression
}

Auf den folgenden Seiten möchte ich mich einem aufsehenerregenden Akt symbolischer Kommunikation in der ausgehenden Republik zuwenden und versuchen, die Signifikanz dieser Szene herauszuarbeiten und die Bedingungen für das Gelingen oder Scheitern einer nonverbalen Kommunikation zu analysieren. Wir befinden uns in der Zeit kurz vor Caesars Ermordung, als den Quellen zufolge die römische Öffentlichkeit von der Diskussion beherrscht wurde, welcher politische Status dem Dictator zukam, ob er noch Teil der republikanischen Ordnung oder nicht doch vielmehr als König oder gar Tyrann anzusprechen und zu behandeln sei. In dieser Phase einer Unentschiedenheit oder eines Schwebezustands ereignete sich etwas Unerhörtes, wie uns Sueton mitteilt: ${ }^{20}$

\begin{abstract}
78 (I) Verum praecipuam et exitiabilem sibi inuidiam hinc maxime mouit. adeuntis se cum plurimis honorificentissimisque decretis uniuersos patres conscriptos sedens pro aede Veneris Genetricis excepit. quidam putant retentum a Cornelio Balbo, cum conaretur assurgere; alii, ne conatum quidem omnino, sed etiam admonentem Gaium Trebatium ut assurgeret minus familiari uultu respexisse. (2) idque factum eius tanto intolerabilius est uisum, quod ipse triumphanti et subsellia tribunicia praeteruehenti sibi unum e collegio Pontium Aquilam non assurrexisse adeo indignatus sit, ut proclamauerit: repete ergo a me Aquila rem publicam tribunus! et nec destiterit per continuos dies quicquam cuiquam nisi sub exceptione polliceri: si tamen per Pontium Aquilam licuerit. 79 (I) adiecit ad tam insignem despecti senatus contumeliam multo arrogantius factum. (Suet. Iul. 78,I-79,I)
\end{abstract}

78 (I) Aber den empfindlichsten und verhängnisvollen Hass zog sich Caesar vor allem durch Folgendes zu: Die Senatoren, die ihn vollzählig mit zahlreichen und außerordentlich ehrerbietigen Beschlüssen aufsuchten, empfing er vor dem Tempel der Venus Genetrix, und zwar sitzend. Einige meinen, er sei von Cornelius Balbus zurückgehalten worden, als er sich dazu entschloss aufzustehen; andere hingegen, er habe nicht einmal den

I9 Von der Lesbarkeit der Körpersprache geht etwa Cicero aus: Cic. nat. deor. 2,I45; off. I,I28; Lael. 88. Vgl. aus späterer Zeit Sen. epist. 52,I2; Ambr. off. I,I8,7I: habitus enim mentis in corporis statu cernitur.

20 Der Vorfall ereignete sich wohl im Dezember des Jahres 45. 
Versuch dazu unternommen, sondern habe sogar, als ihn Gaius Trebatius ermahnte, sich zu erheben, mit einer wenig freundlichen Miene auf ihn geblickt. (2) Diese seine Verhaltensweise schien deshalb um so unerträglicher, als er selbst seine Entrüstung gezeigt habe, dass sich Pontius Aquila als einziges Mitglied des Kollegiums nicht erhoben habe, als er bei einem Triumphzug an den Sitzen der Tribunen vorbeifuhr; Caesar war so empört, dass er ausgerufen haben soll: „Dann fordere du, Aquila, Tribun, von mir das Gemeinwesen zurück!“ Und noch mehrere Tage danach unterließ er es nicht, irgendjemandem ein Versprechen nur mit der Einschränkung zu geben: „Aber nur, sofern Pontius Aquila es gestattet.“ 79 (I) Caesar fügte einer so auffälligen Herabwürdigung des Senats ein weitaus arroganteres Benehmen hinzu. ${ }^{2 \mathrm{I}}$

Wir werden hier Zeugen einer bemerkenswerten sozialen Interaktion im römischen Stadtraum. Die vollständig versammelte Senatorenschaft begibt sich, nachdem sie zahlreiche Ehrungen für Caesar beschlossen hat, über das Forum Iulium zu ebendem Empfänger der Ehren, um ihm diese vor aller Augen anzutragen. Statt sich von seinem Stuhl zu erheben, verharrt Caesar in seiner Position und bringt damit anscheinend eine politische und soziale Relation zum Ausdruck. ${ }^{22}$ Ohne ein einziges Wort zu sagen, signalisiert er, wie er die politische Hierarchie zwischen sich und den Senatoren einschätzt. Er verletzt durch das Sitzen eklatant bestimmte Regeln sozialer Interaktion und stößt seine Interaktionspartner vor den Kopf, wie an dem dadurch ausgelösten Affekt der inuidia abzulesen ist. Da Caesar den Andeutungen Suetons zufolge die Erwartungen der Senatoren ignoriert und gerade der Beginn einer Begegnung üblicherweise bestimmten Regeln folgt und ritualisiert ist, scheint der Dictator eine Norm zu verletzen, ein Ritual, mit dem die Senatoren gerechnet haben, zu stören. Sueton lenkt zusätzlich darauf die Aufmerksamkeit, indem er eine Episode zum Vergleich anführt, in der Caesar selbst das Opfer einer solchen Normentransgression gewesen ist und diese offenkundig als Affront begriffen hat. ${ }^{23}$ Der Dictator hätte, so gibt der Historiograph zu verstehen, dem Senat denselben Respekt zollen müssen, den er selbst vom Volkstribun erwartete. So aber wird sein Verhalten als Hybris betrachtet.

Ihre volle Bedeutung erhält die Regelverletzung bei Sueton freilich erst durch den Kontext. Der Autor reiht in diesem Abschnitt zahlreiche Begebenheiten aneinander, in denen symbolische Kommunikation unter dem Leitthema der Ehre im Mittelpunkt steht. ${ }^{24}$ Der Leser gewinnt den Eindruck, als sei die Zeit unmittelbar vor Caesars Ermordung eine einzige Abfolge von symbolischen Akten gewesen, die zu öffentlichen Diskussionen angeregt hätten. ${ }^{25}$ So ließ sich Caesar nicht allein den Namen Imperator verleihen und akzep-

2I Mit dieser Bemerkung leitet Sueton zu den Vorfällen am Latiner- und am Lupercalienfest über.

$22 \mathrm{Zu}$ der Episode vgl. Jehne (1987) 279f.; Dobesch (I988), dem allerdings mehr an einer Quellenanalyse gelegen ist als an einer Interpretation der Szene.

23 Vgl. dazu Jehne (1987) $23 \mathrm{f}$.

24 Suet. Iul. 76-79. Siehe ferner Nic. Dam. Vit. Caes. 69-75; D.C. 44,3-7. Zu Caesars Erhöhung durch diese exzeptionellen Ehrungen, mit denen sich die Senatoren gegenseitig zu überbieten versuchten, Jehne (I987) I9I-220.

$25 \mathrm{Zu}$ den Caesars Ermordung vorausgehenden Ereignissen siehe jetzt Lintott (2009). 
tierte ein Standbild neben denen der Könige; er duldete es auch, dass man ihm einen goldenen Sessel in der Kurie und vor Gericht einrichtete, Tempel und Bildnisse neben den Göttern widmete, ihm einen eigenen Priester zudachte und einen Monat nach ihm benannte. Er selbst ergriff die Initiative zu symbolischen Handlungen, wenn er noch am 3I. Dezember einen Konsul für wenige Stunden ernannte und die Staatsfinanzen seinem Privatsklaven anvertraute, wodurch er zu verstehen gab, dass er nach eigenem Gutdünken mit dem Staatswesen zu verfahren gedachte. Ja, es kam sogar zu einem regelrechten öffentlichen Agon um Ehrungen, als Caesars Standbild am Latinerfest mit einem Lorbeerkranz gekrönt wurde, die Volkstribunen diesen aber herunterreißen ließen, was Caesars Missfallen erregte. Nach dem Sitzenbleiben vor den Senatoren und der Konfrontation mit den Volkstribunen folgte der dritte Akt des Schauspiels beim Fest der Lupercalien: Mehrmals versuchte der Konsul Antonius, dem Dictator ein Diadem als Zeichen monarchischer Repräsentation aufs Haupt zu setzen, doch verweigerte dieser es jedesmal, indem er das Abzeichen dem Gott Jupiter darbringen ließ. ${ }^{26}$ Indem Sueton die durch eine verdichtende Darstellung geknüpfte Kette dieser Ereignisse ${ }^{27}$ auf Caesars Ermordung hinauslaufen lässt und dabei stets auf die Rezeption durch die stadtrömische Bevölkerung rekurriert, ${ }^{28}$ unterstreicht er, welche Auswirkungen symbolische Akte wie beispielsweise Gesten auf die öffentliche Meinung haben konnten. Die diesen Handlungen und Begebenheiten inhärente Sprengkraft liegt darin, dass mit jeder Ehrung, die Caesar annimmt oder selbst initiiert, das fragile Kräfteverhältnis, auf dem die römische Republik beruht, schrittweise zugunsten der herausragenden Einzelpersönlichkeit verschoben wird. Je außergewöhnlicher die Ehrung und je selbstherrlicher Caesars Handlungsweise, desto weiter entfernt er sich von der überkommenen Ordnung und nähert sich einem Monarchen, ja sogar den Göttern an. Sueton ist nicht entgangen, welche Signifikanz die einzelnen symbolischen Akte für die Stellung des Dictators zur res publica besitzen. ${ }^{29}$

Nun ist Sueton nicht der einzige antike Autor, der uns über Caesars Sitzenbleiben informiert. Mit leichten Variationen begegnen wir dem Regelverstoß ebenso bei Nikolaos von Damaskus, Livius, Plutarch, Appian und Cassius Dio. ${ }^{\circ}$ Ist schon die Dichte der Überlieferung bemerkenswert, so steigert sich die Aufmerksamkeit, wenn wir im Vergleich der Partien sehen, dass sämtliche Autoren die Episode nicht nur beiläufig erwähnen, sondern in extenso und mit großer Detailfülle ausführen, sie also im höchsten Maße für signifikant

26 Siehe auch Nic. Dam. Vit. Caes. 7I-75; Plu. Caes. 6r; App. BC 2,456-458; D.C. 44,II.

27 Tatsächlich lösten die Begebenheiten einander nicht in so rascher Folge ab, wie es Sueton suggeriert. Vgl. die Bemerkung bei D.C. 44,4 ,I.

28 Suet. Iul. 79,2: Gerücht, er strebe nach Königswürde; 79,3: Gerücht der Übersiedelung nach Troja; 80,I: Volk fordert Befreier von der Alleinherrschaft; 8o,2: Sprüche gegen Caesar. Sueton rahmt den ganzen Passus zu den Ehrungen durch die Bemerkungen praegravant tamen cetera facta dictaque eius, ut et abusus dominatione et iure caesus existimetur $(76, \mathrm{I})$ und quae causa coniuratis maturandi fuit destinata negotia $(80, \mathrm{I})$.

29 Vgl. die angeblichen Worte Caesars in Suet. Iul. 77: nihil esse rem publicam, appellationem modo sine corpore ac specie.

30 Nic. Dam. Vit. Caes. 78-80; Liv. perioch. II6; Plu. Caes. 6o; App. BC 2,445-447; D.C. 44,8; Eutr. 6,25. 
halten. Insbesondere gehen sie der Frage nach, aus welchen Gründen Caesar sitzen blieb. Während bei Sueton erwogen wird, der Dictator sei von jemandem aus seiner Umgebung abgehalten worden, wird von Nikolaos, der deutliche Sympathien für Caesar hegt, diskutiert, ob dieser gerade im Gespräch abgelenkt gewesen sei. ${ }^{3 \mathrm{I}}$ Ferner verweist man auf Caesars Epilepsie als möglichen Grund für Unachtsamkeit, ${ }^{2}$ oder bei Cassius Dio finden wir die geradezu aberwitzige und auch sogleich verworfene Erklärung, Durchfall habe Caesar am Aufstehen gehindert.33 In polemischer Brechung sind hier noch die Versuche aus Caesars Umgebung zu greifen, dessen Gebaren in irgendeiner Weise zu rechtfertigen. Was diese Diskussionen evoziert und auch den Leser beschäftigt, ist die zentrale Frage nach Caesars Motiv bzw. nach der Intentionalität der Handlung. Ein mutmaßlich symbolischer Akt wirft in den Augen der Betrachter die Frage auf, ob denn tatsächlich eine Intention vorgelegen hat oder ob kontingente, vorzugsweise körperliche Faktoren das Verhalten des Akteurs erzeugten. 34

Mag man auch zubilligen, dass die weitgehend senatorisch geprägte Tradition der Historiographie die Episode über Gebühr beachtet und den Protagonisten aus der Rückschau in ein ungünstiges Licht rückt, ebenso, dass es übertrieben ist, die Begebenheit als den entscheidenden Ausschlag für die Ermordung anzusehen, 35 so darf man gleichwohl aus dem einhelligen Bericht schließen, dass Caesars Sitzenbleiben bereits bei seinen Zeitgenossen Kopfschütteln, wenn nicht Empörung ausgelöst hat. ${ }^{6}$ Hätte Caesars Verhalten kein Aufsehen erregt, wäre es wohl kaum zu den verschiedenen Erklärungsversuchen gekommen, die sich in der späteren Geschichtsschreibung niedergeschlagen haben. Doch worin lag der Grund für diese Entrüstung? Als Dictator nahm Caesar eine aus der verfassungsgemäßen Ordnung herausgehobene politische Stellung in Rom ein, die ihn von den jährlich wech-

3I Bei Nikolaos ist Caesar so sehr mit dem Bau des Forums beschäftigt und in das Gespräch mit den Handwerkern vertieft, dass er die herannahenden Senatoren nicht bemerkt (Vit. Caes. $78 \mathrm{f}$.). Dagegen spricht gerade die Tatsache, dass Caesar auf dem Stuhl Platz genommen hat, die eher auf die Beschäftigung mit öffentlichen Angelegenheiten hinweist.

32 Bei Plu. Caes. 6o,6-8 bringt Caesar selbst seine Krankheit erst nachträglich als Rechtfertigung vor. Plutarch weist dies jedoch als Ausflucht, die nicht der Wahrheit entspreche, zurück.

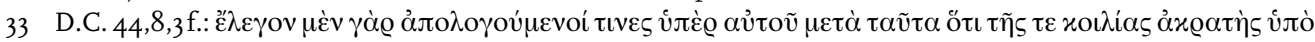

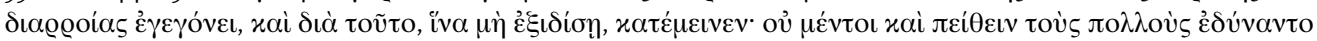

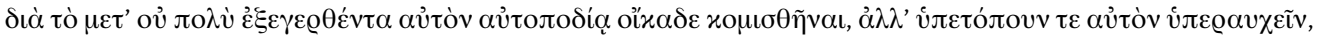

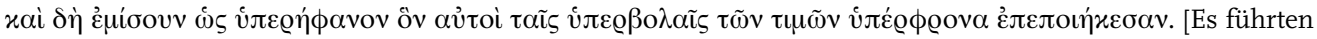
nämlich einige zu seiner Verteidigung im Nachhinein an, er habe wegen Durchfalls keine Gewalt über seinen Leib gehabt und sei sitzen geblieben, damit es nicht zu einer Darmentleerung komme. Indessen vermochten sie die Mehrheit nicht zu überzeugen, da er nach kurzer Zeit aufstand und sich zu Fuß nach Hause begab. Stattdessen vermuteten die meisten, er sei hochmütig, und so hassten sie ihn als einen hochfahrenden Menschen, den sie doch selbst durch das Übermaß der Ehren überheblich gemacht hatten.].

$34 \mathrm{Zu}$ körperlichen Faktoren als möglichen Entschuldigungsgründen für ein Abweichen von sozialen Regeln vgl. auch Quint. inst. II,3,I44.

35 Weinstock (I97I) 275 bewertet die Darstellung der Empörung in den späteren Texten als übertrieben.

36 Neben Suet. Iul. 78, I und 79,I wird die Empörung auch betont von App. BC 2,446; D.C. 44,8,2; 44,8,4; Liv. perioch. II6; Eutr. 6,25. Selbst Nikolaos kann nicht umhin, die Nachwirkung des Sitzenbleibens zuzugestehen (Vit. Caes. 78). 
selnden und kollegial agierenden Magistraten augenfällig unterschied. ${ }^{37}$ Wie diese besaß er selbstverständlich Anspruch auf bestimmte Insignien, also formelle Statussymbole, ${ }^{38}$ und Respektsbezeugungen. So wurden die höheren Beamten von den Lictoren mit Fasces begleitet und waren berechtigt, in der Stadt zu fahren und zu sitzen.39 Zudem hatten die Senatoren, als sie ihm all die genannten monarchischen Ehrungen antrugen, keinen Zweifel daran gelassen, dass sie bereit waren, Caesars exzeptionellen, nachgerade gottgleichen Status zu akzeptieren. Gleichwohl waren sie und die römische Bevölkerung entrüstet, dass der Senat in dieser Weise missachtet, ja verhöhnt wurde.

Zurückzuführen ist die Empörung auf die Implikationen des Sitzens und Stehens, die den Römern in gleicher Weise geläufig waren wie den Angehörigen zahlreicher anderer Kulturen..$^{\circ}$ Ihren sichtbarsten Ausdruck fanden sie in der sella curulis, dem Stuhl, auf dem die Oberbeamten mit Imperium zur Ausübung ihrer Amtsgeschäfte Platz nahmen.4 $\mathrm{Zu}$ allen offiziellen Anlässen, welche dies zuließen, bei der Rechtsprechung, Auspizien und der Aushebung, bedienten sich die curulischen Beamten dieses Stuhls und nahmen ihre Amtsgeschäfte im Sitzen wahr. ${ }^{42}$ Als Abzeichen ihrer Amtsgewalt verkörperte die sella die Würde und die Autorität der Magistratur, so dass sie auch bei Leichenzügen mitgeführt wurde, um an auctoritas und dignitas der Ahnen zu erinnern, oder in der späten Republik zur Selbstdarstellung auf Reliefs dargestellt wurde 43 und zur Propagierung politischer Ideen auf Münzbildern erschien.44 Wie den regulären Beamten, so stand die sella curulis den außerordentlichen Magistraten, dem Interrex und dem Dictator zur Verfügung. ${ }^{45}$ Auch in dieser Hinsicht sprengte Caesar das traditionelle Gefüge, indem er die Auszeichnung erhielt, die sella ständig zu führen, also unabhängig von der Ausübung eines bestimmten Amtes. Überdies wurde sein Sitz vergoldet und erinnerte so unübersehbar an monarchische Throne; durch dieses singuläre Kennzeichen vertiefte sich die Kluft zu den übrigen Beamten. $4^{6} \mathrm{Da}$ das Sitzen die höheren Ämter sinnfällig kennzeichnete, gebot es der Respekt,

37 Siehe Mommsen (I887/8) 2.I,I4I-I72; Jehne (I987) I5-38; Kunkel u. Wittmann (I995) 665-7I9, insbesondere $7 \mathrm{I} 2-7 \mathrm{I} 6$.

38 Der Begriff bei Kolb (I977) 24I.

$39 \mathrm{Zu}$ Insignien und Tracht der römischen Magistrate Mommsen (I887/8) I,372-435; Schäfer (1989); Kunkel u. Wittmann (I995) II9-I23.

$40 \mathrm{Zu}$ Sitzen und Stehen Sittl (I890) I52-I54; Davies (2005); im Hinblick auf Griechenland Bremmer (I992). Ähnlich wie im Sitzen und Stehen konnten sich Status oder Gruppenzugehörigkeit auch in einer bestimmten Art des Ganges manifestieren. Siehe Corbeill (2004) I07-I39.

4I Die sella curulis stand den regulären Ämtern der Konsuln, der Praetoren und der curulischen Aedilen zu, ferner den nichtregulären Ämtern Interrex, Dictator und Magister equitum; hinzu kamen die Decemviri legibus scribundis, die Tribuni militum consulari potestate, die Censoren und der Flamen Dialis. Siehe Mommsen (I887/8) I,399-402; Schäfer (I989) 50-52; Kunkel u. Wittmann (I995) I09.

42 Mommsen (I887/8) I,397-408; Schäfer (I989) 63.

43 Ronke (1987) 256-283.

44 Schäfer (I989) 70-I95.

45 Zur sella curulis des Dictators Liv. 6,I5,I; D.H. Io,24; Lyd. Mag. I,37.

46 Nic. Dam. Vit. Caes. 7I; D.C. 44,6,I; 44,I7,3; App. BC 2,I06; Suet. Iul. 76,I (sedem auream in curia et pro tribunali). Schäfer (1989) II5. 
dass, wer mit dem betreffenden Amtsträger zu tun hatte, seinerseits stand. In der Literatur wird an zahlreichen Stellen beschrieben, wie sich andere Personen zur Ehrerbietung vor den hohen Beamten erheben. 47 Auch vom Senat erwartete man, dass er die Konsuln im Stehen begrüßte. Wer auf einem Pferd ritt oder im Wagen fuhr, musste, sobald er einem Konsul oder Praetor begegnete, absteigen und vor diesem auf dem Erdboden stehen, wozu die Liktoren ausdrücklich aufforderten. $4^{8}$ Noch in der Kaiserzeit, als die republikanischen Ämter längst ihre politische Bedeutung eingebüßt hatten, behielt man diese Gewohnheiten bei, und selbst einige Kaiser ließen es sich nicht nehmen, ihren Respekt gegenüber dem Konsulat auf diese Weise zum Ausdruck zu bringen und damit ein Bekenntnis zur res publica abzulegen. So stand Kaiser Tiberius demonstrativ vor den Konsuln auf.49 Plinius hält in seinem Panegyricus voller Bewunderung fest, dass Trajan vor dem sitzenden Konsul steht, der diese Ehrenbezeugung als etwas völlig Selbstverständliches hinnimmt. ${ }^{\circ}$ Fraglos herrschte in der Kaiserzeit die Erwartung, dass man sich auch vor dem princeps selbst oder dessen Angehörigen erhob.5 ${ }^{\text {I }}$ Bei Akklamationen zeigte die Bevölkerung ihre Zustimmung zum Kaiser durch Beifall an, und zwar im Stehen..$^{52}$ Umgekehrt konnte ein Kaiser seine Bescheidenheit und Leutseligkeit demonstrieren, indem er Magistraten das Sitzen in seiner Gegenwart ausdrücklich gestattete oder wie Augustus die Respektsbezeugung des Aufstehens zumindest für die jüngeren Mitglieder des Kaiserhauses zurückwies.53

In Rom existierten demnach feste Konventionen oder - wie im Falle der republikanischen Magistrate - sogar klare Regeln, wie durch Sitzen und Stehen Würde und Achtung öffentlich zum Ausdruck gebracht werden konnten. Im gleichen Atemzug muss freilich betont werden, dass diese symbolische Körpersprache keineswegs so starr reglementiert war, wie es auf den ersten Blick scheint. Auch wenn das Ausführen von Amtsgeschäften zwingend das Sitzen auf der sella erforderte, gab es in sonstigen sozialen Interaktionen ausreichenden Spielraum für Widerstände oder Abweichungen. Wie eben erwähnt, konnte ein Kaiser durchaus Ausnahmen zulassen. Immer wieder kam es zu Machtproben, wenn jemand das Aufstehen verweigerte, oder wir hören von Vorbehalten gegenüber dem Privileg des Sitzens. ${ }^{54}$ Nicht allein Caesar tat sich hierbei hervor, auch der kynische Philosoph Demetrius stieß den Kaiser Vespasian durch demonstratives Sitzenbleiben vor

47 Cic. Pis. 26; Liv. 9,46,9; Sen. epist. 64,Io; Suet. Claud. ı2. Zum assurgere als Zeichen der Ehrerbietung gegenüber Älteren Cic. Cato 63; inv. I,48; Gell. 2,I5. Procopé (I991) 965-969.

48 Sen. epist. 64,I0; Arnob. nat. 7,I3. Aufforderung der Liktoren: Val. Max. 2,2,4; Plu. Fab. 24; D.C. 36,35 .

49 Suet. Tib. 3I,2.

50 Plin. paneg. 64,2-4.

5I Philostr. Her. I2; Philostr. VA 3,27.

52 Cic. Sest. II5; Suet. Aug. 56; Phaedr. 5,7,25-28; Plin. paneg. 54,3. Vgl. die Parodie darauf in Petron. 6o. Aldrete (1999) 104-108.

53 Suet. Claud. I2; Aug. 56.

54 Liv. 9,46,9; Val. Max. 3,7,II; 8,5,6; Gell. 7,9,5; Suet. Tib. 3I; D.C. 45,I6; 57,II. Cassius Dio berichtet, dass M'. Acilius Glabrio den Befehl gegeben habe, die sella des Praetors L. Licinius Lucullus zu zerstören, weil dieser zuvor nicht vor ihm aufgestanden war (36,4I,2). 
den Kopf.55 Soziale Interaktionen folgen eben nur in den seltensten Fällen schriftlich kodifizierten Regeln; weitaus häufiger beruhen soziale Normen auf Sitte und Herkommen, bilden mithin einen allgemeinen Konsens, der in der Regel nicht herausgefordert wird. Gerade weil sie nicht schriftlich niedergelegt und einklagbar sind, erweisen sie sich als besonders anfällig für Transgressionen. Der Bruch einer sozialen Norm zieht nicht automatisch eine genau festgelegte Sanktion nach sich, vielmehr sind die Reaktionen stark durch den jeweiligen Kontext bedingt. Außerdem bedeutet ihr traditioneller Charakter, dass sie keineswegs faktische Relationen, etwa politische Hierarchien, exakt widerspiegeln. $5^{6} \mathrm{Da}$ solche Konventionen ihrem Wesen nach träge sind, kann es sein, dass sie Verhältnisse abbilden, die längst nicht mehr gegeben sind. Illustriert wird das Beharrungsvermögen sozialer Normen nicht zuletzt durch das Verhalten der Senatoren und der römischen Bevölkerung bei Caesars Sitzenbleiben. Während der Dictator gegenüber den Senatoren eine Haltung einnimmt, welche die tatsächlichen Machtverhältnisse visualisiert, rechnen die patres conscripti immer noch damit, dass er sich den Gepflogenheiten entsprechend verhält, also ihnen als dem zentralen Organ der römischen Republik den gebührenden Respekt zollt. Wenn Regeln verletzt worden waren, so offenbar nicht die des römischen Staatsrechts, sondern andere, ungeschriebene. Die Bedeutung des demonstrativen Sitzens wird durch den positivistischen staatsrechtlichen Diskurs nicht zureichend erfasst,57 insofern das Sitzen immer eine soziale Dimension besitzt, die nicht in juristischen Kategorien zu greifen ist..$^{8}$

\section{Interaktion auf der Bühne}

Nun tragen symbolische Akte per definitionem unter der oberflächlichen eine oder mehrere weitere, verborgene Bedeutungen. Sie verweisen als zeichenhafte Handlungen auf etwas, geben mehr zu verstehen, als sichtbar wird. Eine eingehendere Analyse des Kommunikationsprozesses vermag die verschiedenen Bedeutungsebenen aufzudecken, so dass noch klarer zum Vorschein kommt, weswegen die Senatoren empört reagieren. In erster Linie sind an ihm beteiligt der Akteur Caesar und der Empfänger der Handlung, also die Gruppe der Senatoren. An politischer Signifikanz gewinnt die Szene dadurch, dass die Senatoren von den beiden Konsuln und weiteren Beamten mit Liktoren begleitet werden.59

55 Suet. Vesp. I3.

56 Dies zeigt sich etwa auch in Ritualen und Verhaltensweisen, die gegenüber dem einfachen Volk die Jovialität, aber auch den Respekt der römischen Aristokratie zur Schau stellen sollten. Siehe Jehne (2000).

57 So hingegen Weinstock (I97I) $275 \mathrm{f}$.

58 Sehr schön beleuchtet dies die bei Gell. 2,2,I-II überlieferte Begebenheit, bei der es zu einer Diskussion kommt, ob der in dieser Situation einzig verfügbare Sitzplatz einem Vater als Privatperson aufgrund seines Alters zustehe oder dem Sohn in seiner Eigenschaft als Provinzstatthalter. Es kollidieren also eine soziale und eine staatsrechtliche Norm.

59 So Nic. Dam. Vit. Caes. 78. 
Indem Caesar sitzen bleibt und damit die Erwartungen der Senatoren und Magistrate durchbricht, vollzieht er an ihnen eine Handlung und manipuliert sie. Sueton erweckt durch sein Schweigen den Eindruck, als seien sie so konsterniert, dass nicht nur von den Ehrungen, die sie ihm eigentlich antragen wollen, nicht mehr die Rede ist, sondern sie auch den Rückzug antreten, ohne auf Caesars nonverbale Kommunikation etwas zu entgegnen. Mit seiner Brüskierung und Demütigung scheint der Dictator Erfolg zu haben. Indes beschränkt sich die Kommunikation nicht auf die genannten Beteiligten, vielmehr bedarf es eines Dritten, damit die von Caesar mutmaßlich intendierte Botschaft ihre volle Wirkung entfaltet. Wären die Senatoren unter sich in der Kurie mit Caesars anmaßendem Verhalten konfrontiert gewesen, so hätte dies gewiss gleichermaßen für Empörung gesorgt, doch hätte die römische Öffentlichkeit nicht unbedingt davon erfahren. Vollkommen wird der Gesichtsverlust der patres conscripti erst dadurch, dass die Interaktion beobachtet wird. Wenn Caesar das Sitzenbleiben im voraus geplant hat, so hat er zweifelsohne ein weiteres Publikum im Blick gehabt, vor dessen Augen sich die Demütigung abspielen sollte. Ohne dass Sueton dies eigens erwähnt, kann man voraussetzen, dass bei einem derart aufsehenerregenden Vorgang wie dem Zug des ganzen Senats zum Tempel zahlreiche Menschen auf dem Platz des Forums zugegen gewesen sind. ${ }^{60}$ Dieses weitere Publikum muss jedoch nicht unbedingt zur Gänze dem Ereignis beiwohnen, sondern kann auch später, etwa durch Hörensagen, davon erfahren. Auch im eingangs erwähnten Falle des israelischen Vizeaußenministers ist die Botschaft medial vermittelt. Wir haben folglich eine Dreiecksbeziehung zwischen einem Akteur, einem Rezipienten und einem Publikum vor uns, wobei dieses Publikum mindestens in gleichem Maße Adressat der Botschaft ist wie der primäre Rezipient. Die Beobachter der Interaktion sind konstitutiv für das Gelingen des symbolischen Kommunikationsaktes. Denn nur wenn eine gewisse Form von Öffentlichkeit gewährleistet ist, kann von einer wirklichen Demütigung oder einem Gesichtsverlust die Rede sein.

Das lenkt die Aufmerksamkeit darauf, dass symbolische Kommunikation eine soziale Dimension besitzt. Wer eine symbolische Handlung vollzieht, legt es darauf an, von einer Öffentlichkeit beobachtet zu werden; andernfalls verpuffte die beabsichtigte Wirkung. Man könnte mithin von einer Aufführung vor Zuschauern oder von einem ,sozialen Drama‘ sprechen, in dem es eindeutig definierte Rollen gibt und das offensichtlich vom Regisseur Caesar inszeniert wird. ${ }^{6}$ Ohne dass die Senatoren damit rechnen bzw. nach einem ande-

60 Nur Nic. Dam. Vit. Caes. 78 erwähnt, dass dem Zug der Senatoren eine Volksmenge gefolgt sei.

6I Unter einem sozialen Drama ist nach Turner (I989) IO-I2, I08-I39 ein Prozess zu verstehen, bei dem der Bruch einer sozialen Norm zu einer Krise führt, die durch verschiedene mögliche Maßnahmen bewältigt wird, ehe es schließlich zu einer Reintegration oder der vollkommenen Spaltung der beteiligten Gruppe kommt. Es handelt sich meist um politische Prozesse mit einer universellen Verlaufsform, die der aristotelischen Beschreibung der Tragödie ähnelt. Turner postuliert ein Verlaufsschema solcher Dramen mit mehreren Stadien (ebd. IIf., I08-II2). Obgleich dieses Modell den Vorgängen vor Caesars Tod nicht ganz entspricht, lassen sich auch hier verschiedene Stadien bzw. ,Akte‘ differenzieren: (I) Caesar verletzt die Regel der gegenseitigen Gesichtswahrung. 
ren Drehbuch, als sie es erwarten, weist der Dictator ihnen den Part eines Darstellers zu, der dem Agieren des Protagonisten passiv ausgeliefert ist und nur den Handlungspielraum zur Verfügung hat, den der Regisseur ihm zubilligt. Dem Publikum signalisiert die Szene, dass die Rollen und damit die politische Macht ungleich verteilt sind. Eine Hierarchie wird eindringlich vor Augen geführt.

Wie die Inszenierung eines Dramas einer adäquaten Bühne bedarf, so ist auch in unserem Beispiel der Raum, in dem sich die nonverbale Kommunikation vollzieht, nicht unerheblich. Da die Sichtbarkeit des Aktes wie gesagt von entscheidender Bedeutung ist, muss sich das Drama an einem öffentlich zugänglichen Ort abspielen, nicht etwa in einem den Augen der Menge entzogenen Privatraum. Die Szenerie könnte von dem in Personalunion beteiligten Regisseur und Protagonisten Caesar nicht passender gewählt sein. Schon dass die Senatoren sich von ihrem eigenen Raum, der Kurie, zu ihm aufmachen müssen, um ihm die Ehren anzutragen, impliziert eine asymmetrische Relation zwischen den Beteiligten. ${ }^{62}$ Noch dazu ist es nicht irgendein zufälliger Ort, an dem Caesar die Senatorenschaft zu empfangen geruht, sondern der im Jahre 46 geweihte Tempel der Venus Genetrix, der julischen Stammutter, auf dem von Caesar selbst geschaffenen Forum (Abb. 2). Der Dictator gestaltet also einerseits das Stadtzentrum nach seinen Vorstellungen, indem er die Curia Iulia auf dem Platz des Comitiums errichten lässt, die Rostra auf die Westseite des Forum Romanum verlagert, die Basilica Iulia in Auftrag gibt und schließlich das Forum Iulium anlegt. Gerade die städtebauliche Lage und Form dieses Forums machten, obgleich es bei Caesars Tod noch nicht zur Vollendung gediehen war, jedem Römer deutlich, dass ein groß dimensionierter Konkurrenzraum zum politischen Zentrum der Stadt und des Reiches intendiert war. ${ }^{63}$ Deutlicher ließ sich kaum zum Ausdruck bringen, dass Caesar nicht gewillt war, wieder ins Glied zurückzutreten und die überkommene politische Ordnung zu akzeptieren. ${ }^{64}$ Andererseits diktiert er gewissermaßen die Bewegungen in diesem Raum, indem er durch seinen Aufenthaltsort die Senatoren zwingt, sich über das Caesar-Forum zu ihm zu begeben. Nachdem sie die Platzanlage durch den Zugang an der Südostecke betreten haben, müssen sie das gesamte Forum durchschreiten, bis sie am

(2) Der Konflikt schaukelt sich hoch; allgemeine Empörung, verstärkt durch weitere Begebenheiten. (3) Die Gegner planen und ergreifen gewalttätige Maßnahmen. (4) Vernichtung des Störfaktors Caesar.

62 Genauere Angaben, wo der Senat getagt hatte, werden in den Quellen nicht gemacht. Denkbar wäre also auch ein anderer Sitzungsort.

63 Das Forum besitzt eine Ausdehnung von etwa I60 auf $75 \mathrm{~m}$. Appian betont, dass es sich nicht um einen Marktplatz, sondern um einen öffentlichen Raum für Versammlungen handelte (App. BC 2,IO2). Bereits im Jahre 54 war Cicero für Caesar mit dem Erwerb des Landes befasst (Cic. Att. 4,I7). Für das Jahr 52 berichtet dann Suet. Iul. 26 von Baumaßnahmen. Nach dem Sieg über Gallien wurde es am 26. 09. 46 eingeweiht, doch zog sich die Vollendung bis in die Zeit des Augustus hin. Die heute sichtbaren aufrechten Überreste stammen von Erneuerungen der Zeit Trajans und der Spätantike, doch gehen die Dimensionen auf die ursprüngliche Gestalt zurück. Auf dem Platz des Forums befand sich eine Reiterstatue Caesars (Plin. nat. 8,154f.; Suet. Iul. 6I). Amici (I99i), bes. 29-58; Ulrich (1993); Morselli (1995); Stamper (2005) 92-102.

64 Cassius Dio bezeichnet den Tempel der Venus Genetrix nicht unpassend als ein Heroon für Caesar (D.C. 5I,22,4). 


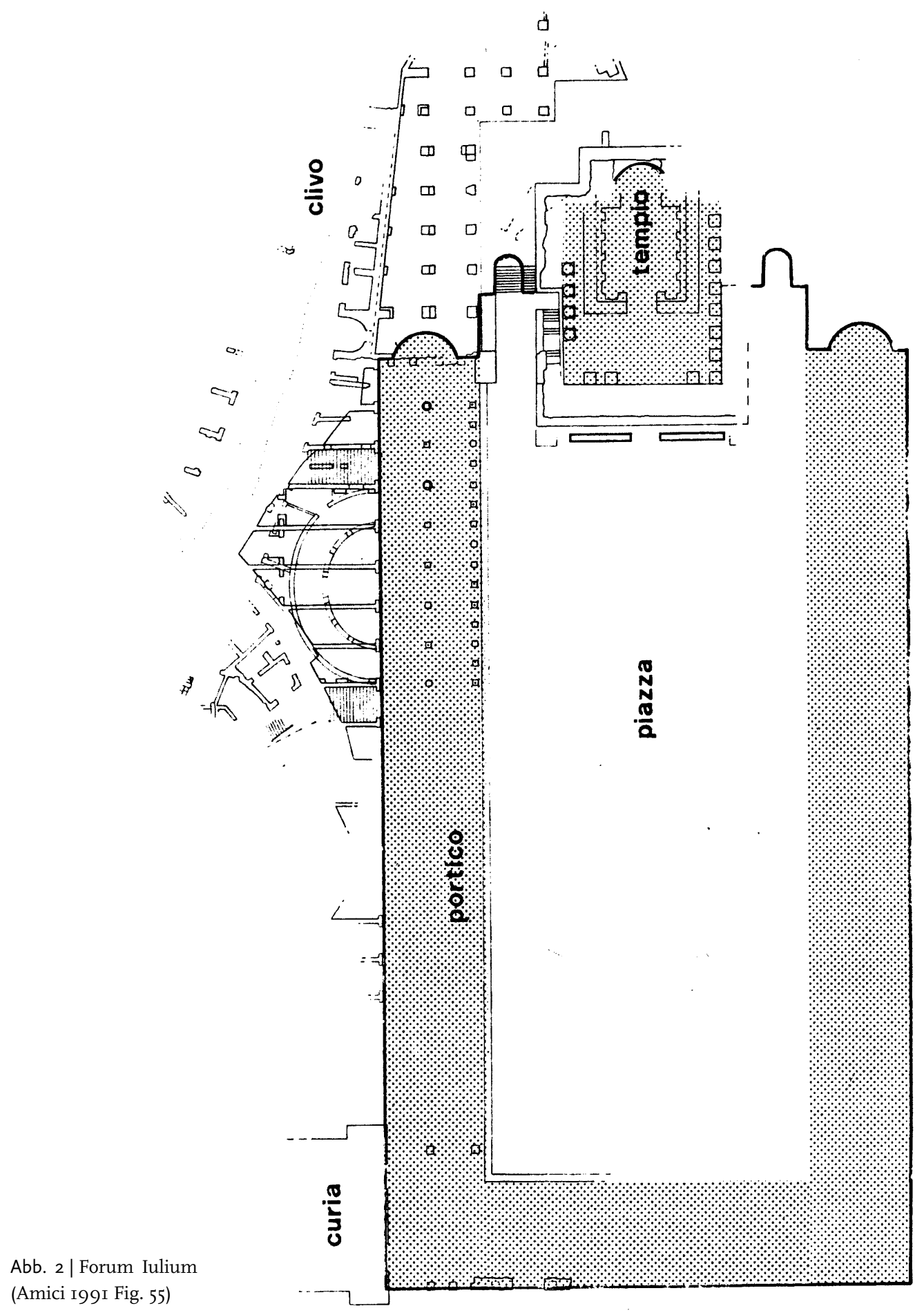


Tempel anlangen, wo sich der Dictator aufhält. Folgerichtig müssen sie sich nach der Brüskierung wieder aus Caesars Sphäre zurückziehen, als wäre eine ungünstig verlaufene Audienz oder eine Huldigung im Herrscherpalast beendet.

Überdies müssen wir uns vor Augen halten, dass das Forum nicht nur auf den Tempel der Venus Genetrix als Fluchtpunkt ausgerichtet war, sondern dieser selbstverständlich durch einen Sockel über das übrige Platzniveau erhoben wurde und somit die ganze Anlage dominierte. ${ }^{65}$ Vor dem eigentlichen Tempelbau erstreckte sich über die volle Breite eine im ursprünglichen Zustand etwa $2 \mathrm{~m}$ tiefe Plattform, die an den beiden Längsseiten des Tempels von hinten her durch Treppen zugänglich war. Sie besaß eine Höhe von etwa 3,5 $\mathrm{m}$ und wurde ihrerseits um $2 \mathrm{~m}$ von dem Bodenniveau des Pronaos übertroffen, so dass die Bodenfläche des Tempels beinahe $6 \mathrm{~m}$ über dem Forumsplatz lag. Man hat in der archäologischen Forschung erwogen, ob nicht der Sockel des Tempels sogar als Rednertribüne konzipiert gewesen sei, wodurch dem Heiligtum auch eine politische Funktion zugeschrieben worden wäre. ${ }^{66}$ Die von Caesar intendierte Konkurrenz zur politischen Rolle des Forum Romanum wäre in diesem Falle noch betont worden. Aber selbst wenn dies nicht der Fall war - für eine tatsächliche Benutzung der Plattform für Reden gibt es keine Belege -, ${ }^{67}$ waren die Senatoren gezwungen, zu Caesar eine Treppe hinaufzugehen, um an ihm vorbeizudefilieren. Vermutlich saß Caesar nicht auf der Plattform selbst, ${ }^{68}$ sondern, wie es der Bericht bei Cassius Dio anzudeuten scheint, im Tempel, also wohl im mittleren Intercolumnium, während die patres conscripti ihm zu Füßen auf der schmalen Plattform standen. ${ }^{69}$ Um mitspielen zu können, mussten sie für ihren Auftritt erst einmal die Bühne erklimmen. Wahrscheinlich hatten die Senatoren sogar das Gefühl, sie würden sich in ehrerbietiger Prozession der im Tempel präsenten Gottheit nähern, als sie auf den erhaben thronenden Caesar zugingen. In dieser Weise sollte sich später dann Kaiser Caligula im Castor- und Polluxtempel inszenieren, den er zum Eingang seines Palastes umgestaltete. ${ }^{\circ}$ Auch wenn man in Rechnung stellen muss, dass Forum und Tempel zum Zeitpunkt des Schauspiels noch ihrer Vollendung harrten und mit Sicherheit keinen so imposanten Eindruck machten wie in der Kaiserzeit, konnte sich gewiss kein Beobachter der suggestiven Kraft dieser Szene entziehen. Der Vorgang muss jedenfalls für alle auf dem Forum Anwe-

65 Ulrich (1994) II7-I55; Stamper (2005) 93-97; Strocka (2005).

66 Ulrich (I994) II7-I55 sieht den Tempel als einen Vertreter des von ihm als templum rostratum bezeichneten Typus, bei dem ein Kultbau mit einer Rednertribüne kombiniert ist. Kennzeichen dieser Tempel ist, dass sie nicht in einem eigenen sakralen Bezirk liegen, sondern Teil einer Forumsanlage sind und dass die Cella allein über die Tribüne, die unterhalb des Podiums liegt, zu betreten ist (ebd. 9-19).

67 Strocka (2005) gegen Ulrich (1994). Strocka sieht die Plattform primär als ästhetisches Mittel, um die Bedeutung des Forums hervorzuheben. Der von Ulrich postulierte Typus des templum rostratum sei eine Fiktion.

68 So die Vermutung von Ulrich (I994) I32f.

69 Gros (I995) 307; Strocka (2005) I54. Suet. Iul. 78, I spricht nicht allzu präzise von pro aede Veneris Genetricis. D.C. 44,8,I (im Pronaos des Tempels); Liv. perioch. Ii6 (ante aedem).

70 Suet. Cal. 22,2: [Caligula] aede Castoris et Pollucis in uestibulum transfigurata, consistens saepe inter fratres deos, medium adorandum se adeuntibus exhibebat; et quidam eum Latiarem Iouem consalutarunt. 
senden gut sichtbar gewesen sein. Caesar dürfte also die räumlichen Gegebenheiten in seine Inszenierung sorgfältig einkalkuliert haben und somit nicht allein seinem Handeln, sondern auch dem von ihm umgestalteten städtischen Raum eine symbolische Funktion zugedacht haben. ${ }^{7}$ Die Szenerie sowie das Requisit des Stuhls fungierten nachgerade als Mitspieler des Dramas.

Worin besteht nun genau die Interaktion auf dieser umsichtig bereiteten öffentlichen Bühne? Wenn wir davon ausgehen, dass es sich um einen Kommunikationsvorgang, wenn auch einen nonverbalen, handelt, so können wir voraussetzen, dass dieser ähnlichen Regeln unterliegt wie verbale Kommunikation. In Anlehnung an Paul Grices Konversationsmaximen kann man hierbei von einem Kooperationsprinzip sprechen, demzufolge der Modus des Kommunikationsaktes entscheidend zum Gelingen des Austausches beiträgt. ${ }^{2}$ Teil der Botschaft ist demnach nicht allein, was mitgeteilt wird, sondern auch, in welcher Art und Weise dies geschieht. Sofern nicht gewichtige Gründe dagegen sprechen, gehen die Beteiligten davon aus, dass beide Seiten darauf bedacht sind, das Gesicht des Gegenübers nicht zu verletzen, so wie sie auch ihr eigenes Gesicht nicht verlieren wollen.73 Unter dem ,Gesicht' ist in diesem Zusammenhang die Zuschreibung von Eigenschaften durch das Individuum an sich selbst zu verstehen, die das Selbstbild konstituieren; kommt es zu einer sozialen Begegnung, ist der einzelne bestrebt, dieses Selbstbild durch sein Verhalten und Handeln aufrechtzuerhalten, und fühlt sich bestätigt, wenn seine Interaktionspartner dieses Bild von ihm übernehmen.74 Damit Gesichtsverletzungen vermieden werden, existieren in allen Kulturen Konventionen der Höflichkeit, formalisierte Regeln der Gesichtswahrung gewissermaßen, die den Beteiligten Sicherheit geben, selbst wenn sie einander zum ersten Mal begegnen. Nur wenn die Interaktionspartner die Bedürfnisse des jeweils anderen in ihre Überlegungen einbeziehen, also mit Fingerspitzengefühl und Geschicklichkeit agieren, ist gewährleistet, dass sie beide von der Kommunikation einen Nutzen haben. Der Nutzen ist also ein Resultat einer kooperativen Aushandlung und insofern ein Kompromiss. So erwarten die Senatoren, wenn sie Caesar die exzeptionellen Ehrungen antragen, von ihm im Gegenzug eine gewisse Höflichkeit und Respekt; sie setzen trotz des faktisch asymmetrischen Verhältnisses eine Reziprozität von Gaben voraus, da sie der Sitte und dem Herkommen vertrauen. Auch einem Amtsinhaber mit unumschränkter Macht steht nach ihrem Verständnis eine gewisse Bescheidenheit an.

7I Mit apologetischer Tendenz betont hingegen Nikolaos mehrfach die angebliche Arglosigkeit und Unerfahrenheit Caesars in politischen Ränkespielen (Vit. Caes. 59; 67).

72 Grice (I979).

73 Goffman (I986) I5-I7.

74 Goffman (I986) Io spricht hierbei vom image: „Der Terminus Image kann als der positive soziale Wert definiert werden, den man für sich durch die Verhaltensstrategie erwirbt, von der die anderen annehmen, man verfolge sie in einer bestimmten Interaktion. Image ist ein in Termini sozial anerkannter Eigenschaften umschriebenes Selbstbild, - ein Bild, das die anderen übernehmen können.“ (Hervorhebung im Original). 
Caesar hingegen missachtet das Kooperationsprinzip, da er sich nicht mit dem geringeren Nutzen, der aus dem Kompromiss hervorgeht, zufrieden geben will, sondern auf seinen persönlichen Maximalnutzen erpicht ist. Dieser lässt sich jedoch nur auf dem Wege der Konfrontation erzielen, wenn man die Bedürfnisse des anderen ignoriert und gegen dessen Interessen verstößt. Solche zwischen Kooperation und Konfrontation verlaufenden politischen Prozesse werden im Übrigen in der Politikwissenschaft mit Rekurs auf die Spieltheorie analysiert, was darauf aufmerksam macht, dass hier ein soziales Spiel vorliegt, das gewissen Regeln gehorcht und bei dem es darauf ankommt, in der Interaktion mit den Mitspielern bestimmte Positionen auf dem Spielfeld zu besetzen.75 Caesar trachtet danach, die Spielregeln einseitig zu seinen Gunsten zu modifizieren, um sich eine günstige Position zu verschaffen. Er gefährdet die stets prekäre Ordnung der politischen Kommunikation und bringt dadurch die Regeln und ihre Fragilität erst zu Bewusstsein. Solange sich alle Beteiligten normenkonform verhalten, wird die Norm nicht wahrgenommen und problematisiert. Nur wenn sich ein Regelverstoß ereignet, wird die Ordnung selbst zum Thema. Dem Regelverstoß wohnt demnach ein Zug der Selbstreferentialität inne, insofern er die Mechanismen der Regel offen legt und zum Gegenstand der Wahrnehmung macht.

Die Ordnung, die durch das Sitzenbleiben gestört wird, besteht in dem Verhältnis zwischen der Gemeinschaft und dem herausragenden Individuum. Eben deshalb stellt Sueton die Episode in den Kontext all der Ehrungen, die Caesar aus der verfassungsmäßigen Ordnung herausheben. Wie wir den öffentlichen Diskussionen über die Angemessenheit des Königstitels entnehmen können, regt jede weitere Ehrenbezeugung zur Reflexion darüber an, ob sich Caesar noch in die Gemeinschaft einfüge oder nicht. Er selbst tritt durch die Zurückweisung von Auszeichnungen nach Kräften dem Eindruck entgegen, er strebe nach der Königswürde, andererseits erweckt er mit jedem symbolischen Akt der recusatio noch mehr den Verdacht, er habe es genau auf solche Erhöhungen abgesehen. Und nun, beim Empfang der Senatoren und Magistrate vor dem Tempel der Venus Genetrix, gibt Caesar gewissermaßen nach und signalisiert durch sein Sitzenbleiben, dass er nach eigener Überzeugung die übermenschlichen Ehrungen nicht nur nicht ablehne, sondern ganz und gar verdient habe. Das Verharren auf dem Stuhl antizipiert die Auszeichnungen, welche die Senatoren zwar beschlossen, aber noch nicht förmlich verliehen haben. Dadurch aber bringt er die von den Senatoren intendierte Inszenierung des Aktes durcheinander, zeigt ihnen, wer der Regisseur ist und welche Rolle ihnen zugedacht ist. Caesar missachtet die vom Anstand geforderte Pause bzw. das Zögern, ehe man eine Gabe annimmt, und eignet sich die Gabe an, bevor sie überhaupt offeriert worden ist. So konstituiert er sein Gesicht auf Kosten des Gesichts der anderen, die jäh überrascht und in völliger Hilflosigkeit zur Passivität verdammt sind. Er demonstriert vor dem römischen Volk, dass er als göttlich ver-

75 Auch Goffman (I986) bedient sich bei seinen Analysen mehrfach der Metaphorik des Spiels, wie es im Paradigma der Theatralität angelegt ist. 
ehrter Herrscher an der Spitze des Staatswesens sitzt, während Senat und Beamte als seine Untertanen zu betrachten sind..$^{6}$ Hätte er mit seiner Strategie auf lange Sicht Erfolg gehabt, wäre durch diesen Akt eine neue soziale Norm etabliert worden.77

\section{Gesichtsverletzung, Intentionalität und kognitive Kompetenz}

Wir haben bereits bemerkt, dass in den antiken Zeugnissen zu Caesars Sitzenbleiben neben der Entrüstung der Senatoren und der Bevölkerung der Frage der Intentionalität besondere Aufmerksamkeit geschenkt wird, offenbar in der Annahme, dass von ihr die Bewertung des Vorfalls abhängt. Um die Relevanz dieser Frage deutlich zu benennen, sei en passant eine andere Episode in Erinnerung gerufen, der eine ähnliche Signifikanz eignet. Als auf der Griechenlandreise Neros im Jahre 66 der spätere Kaiser Vespasian während der Gesangsdarbietungen des Princeps wiederholt vom Schlaf übermannt wurde, fiel er vollständig in Ungnade und wurde nicht nur aus Neros näherer Umgebung, sondern auch von den öffentlichen Empfängen bei Hofe ausgeschlossen. Gesellschaftlich und politisch isoliert, zog sich Vespasian in eine abgelegene, kleine Stadt zurück, weil er die härtesten Strafen gewärtigte. $7^{8}$ Auch in diesem Falle wurde gegen Regeln der sozialen Interaktion verstoßen. Selbstverständlich gebot es der Respekt vor dem Kaiser, auch noch den scheußlichsten Gesängen mit Aufmerksamkeit und einem Hochgefühl der Wonne zu lauschen, doch gab Vespasian einem natürlichen Bedürfnis nach und schlief ein. Wenn man davon ausgeht, dass dieses Einschlafen nicht intendiert war - Vespasian konnte sich die Konsequenzen ausmalen -, so liegt hier unzweifelhaft vor, was wir als Fehltritt oder Fauxpas bezeichnen würden. Im engeren Sinne bedeutet dies nach Peter von Moos „die unabsichtliche Fehlanwendung einer grundsätzlich akzeptierten und bekannten Regel eines gruppeninternen Codes durch ein zugehöriges Individuum“.79 Kennzeichen des Fehltritts ist es, dass er die Persönlichkeit des Akteurs in der Eigen- und der Fremdwahrnehmung beschädigt. Verletzt werden bei einem Fauxpas ungeschriebene, aber allgemein anerkannte Gesetze, und es ist unabdingbar, dass sich der Fehltritt vor einem Publikum ereignet, also beobachtet wird. Vespasians Einschlafen fällt nur insofern etwas aus dem Rahmen, als der klassische Fauxpas ein einmaliges, unvorhergesehenes Ereignis ist, nicht mehrmals nacheinander auftritt. Wenn jemand vor Zuschauern einen unbedachten Schritt tut und Anstoß erregt, so beeinträchtigt er zum einen das Bild, das er selbst von sich und seinem sozialen

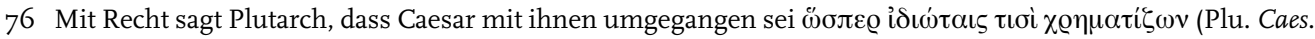
$60,4)$. Dobesch (I988) $42 \mathrm{f}$.

77 Nikolaos rechtfertigt im Übrigen nachträglich Caesars Sitzenbleiben, indem er im Kontext der Ermordung hervorhebt, dass sich der Senat bei Caesars Eintreten zum Zeichen der Ehrerbietung erhoben habe (Vit. Caes. 88). Dies auch bei Plu. Caes. 66,5.

78 Suet. Vesp. 4,4; Tac. ann. I6,5,3. Vgl. Bartsch (I994) 6f. zu den theatralen Aspekten des Vorfalls; ferner Stenger (2010) 397-400.

79 Von Moos (200I) XIV. 
Status besitzt, was zumeist Symptome der Scham hervortreibt, zum anderen beschädigt er aber auch das Bild, das sich die anderen von ihm gemacht haben, da jedermann sieht, dass er zumindest kurzfristig nicht über die Kompetenz verfügt, sich regelkonform in der Öffentlichkeit zu bewegen. Je nach der Schwere des Fehltritts folgen gesellschaftliche Sanktionen, etwa das Vermeiden weiterer sozialer Kontakte. Der Betroffene hat dann sein Gesicht verloren. Wie der Vorfall auf Neros Griechenlandtournee, aber auch manch andere Begebenheit lehrt, war den Römern das Konzept des Fehltritts oder Fauxpas gut vertraut, mochten sie auch nicht über einen eigenen Begriff dafür verfügen..$^{\circ}$

Vespasians Ungeschicklichkeit ist in mehrfacher Hinsicht aufschlussreich, illustriert sie doch eindrücklich, wie in der Interaktion zwischen dem Kaiser und seinen Untertanen alle Beteiligten gewissermaßen Theater spielen, Rollen verkörpern, wobei sie wechselseitig im selben Augenblick beobachtete Akteure sind und Zuschauer. Auch in diesem Theaterstück werden Machthierarchien auf die Bühne gebracht, und von den Beteiligten wird erwartet, dass sie durch rollenkonformes Verhalten die ihnen zugedachte Position einnehmen. Vespasians Einschlafen droht Neros Gesicht zu verletzen, da der Kaiser einen Anspruch auf ungeteilte Aufmerksamkeit und Respekt hat, und wirft so die Frage nach der Macht auf. Da er die labile Ordnung empfindlich stört, muss Vespasian mit Sanktionen rechnen, welche ebendiese Ordnung wiederherstellen sollen. Die Gefährdung von Neros Selbstbild wird dadurch beantwortet, dass Vespasian selbst einen Gesichtsverlust hinnehmen muss. In der sozialen Interaktion stehen mitunter die Selbstbilder mehrerer Beteiligter auf dem Spiel und hängen wechselseitig voneinander ab. ${ }^{81}$

Auf den ersten Blick scheinen Caesars Sitzenbleiben und Vespasians Einschlafen ähnliche Phänomene zu sein, insofern beide Persönlichkeiten vor einem Publikum gesellschaftliche Normen verletzen. Und doch ist das Sitzenbleiben grundsätzlich anders zu bewerten, was mit der Frage der Intentionalität verknüpft ist. Beide Ereignisse ähneln einander, insofern wenigstens einer der Beteiligten die Verhaltensweise eines anderen als verletzend, unfreundlich oder zumindest unhöflich bewertet. Unhöflichkeit existiert freilich nicht, wo nur ein Beteiligter die Gesichtsverletzung intendiert oder wahrnimmt, aber nicht beide bzw. außerdem das anwesende Publikum. Man kann, wenn man das Modell möglicher Gesichtsverletzungen der Übersichtlichkeit halber auf zwei Interaktionspartner reduziert, drei Typen differenzieren: ${ }^{82}$

80 Siehe beispielsweise Plu. 508a (De garrulitate); Val. Max. 7,5,2. Ein etwas anderer Fehltritt liegt in Ovids error bzw. crimen vor, insofern der Dichter anscheinend zur falschen Zeit am falschen Ort etwas gesehen hat, was er nicht hätte sehen dürfen. Die für den Fehltritt konstitutive Öffentlichkeit wurde hierbei eigentlich erst durch die Verbannung und Ovids poetische Verarbeitung hergestellt (Ov. Pont. I,6,20; 2,4,32; 2,6,5; 3,9; trist. 2,IO3f.). Cicero bezeichnet, was unserem Fehltritt gleichkommt, als peccatum stultitiae und gibt eine charakteristische Beschreibung (Cic. de orat. I,II9-I25). Das römische Recht kennt den Begriff der imprudentia für unbeabsichtigte Straftaten, die auf Unachtsamkeit oder Fahrlässigkeit beruhen. Gell. 20,I; Dig. 2,2; Paul. sent. 5,23,3; Serv. ecl. 4,43. 8I Vgl. Goffman (I986) 58.

82 Vgl. Goffman (I986) 20. 
(I) Die Bedrohung des Gesichts ist nicht intendiert:

a. Die Beteiligten bemerken sie nicht oder tun so, als ob sie sie nicht bemerkten. Die soziale Interaktion wird durch den Vorfall nicht weiter gestört.

b. Die Beteiligten nehmen wahr, dass der Akteur sie gerne vermieden hätte. Es handelt sich um einen Fauxpas oder Fehltritt, und der Akteur beschädigt dadurch sein eigenes Gesicht, das heißt, die Gesichtsverletzung fällt auf ihn zurück.

(2) Die Bedrohung des Gesichts ist intendiert:

a. Der Rezipient nimmt diese Intention wahr. Der unfreundliche Akt ist erfolgreich realisiert.

b. Der Rezipient bemerkt dies nicht. Der Versuch bleibt erfolglos. ${ }^{83}$

(3) Die Gesichtsverletzung ist zwar nicht intendiert, geschieht aber als Nebenprodukt einer Handlung und wird in Kauf genommen. Es liegt eine akzidentelle Gesichtsverletzung vor. Für den weiteren Verlauf der Interaktion hängt hier viel davon ab, ob der Rezipient der Handlung eine Intention unterstellt oder nicht.

Es kommt, wie dieser Typologie zu entnehmen ist, in hohem Grade auf die Intention des Akteurs an, was die Frage aufwirft, wie man diese Intention zuschreiben kann. Der Beobachter, in unserem Falle die römische Öffentlichkeit, ist aufgefordert, die Absichten des Akteurs weniger ausfindig zu machen als vielmehr zu rekonstruieren, wobei er verschiedene Faktoren berücksichtigen muss. Ob er dem Akteur eine Intention zuschreibt oder nicht, hängt davon ab, wie er vergangene Handlungen der Kommunikationspartner beurteilt, wie er die sozialen und die Diskursrollen der Beteiligten, den Kontext und das Verhältnis zwischen den Beteiligten einschätzt. Außerdem gehen selbstverständlich Sympathien und Antipathien in die Zuschreibung ein, das heißt, der Akt wird immer in seinem Kontext bewertet, besitzt eine Vorgeschichte. Selbst wenn Vespasian gänzlich ohne Hintergedanken eingeschlafen sein sollte, erachtet Nero dies als Bedrohung seines eigenen Gesichts und geht davon aus, dass Vespasian sie zumindest in Kauf genommen habe. Allein dies genügt als Zeichen mangelnden Respekts.

Caesars Sitzenbleiben scheint auf den ersten Blick keine Frage offen zu lassen. Die Diskussionen und divergierenden Erklärungen, die bei Sueton und den anderen Autoren ihren Niederschlag gefunden haben, zeigen uns jedoch, dass die symbolische Kommunikation zwar den Vorteil einer visuellen Eindrücklichkeit bietet, aber diese auf Kosten der Eindeutigkeit erkauft. Weil ihre Beweggründe nicht offen zutage liegen, bietet sie Ansätze für mannigfaltige Interpretationen. Wenn wir der Einschätzung Suetons und anderer Glauben schenken dürfen, so setzt sich aber schließlich bei Senat und Volk die Meinung durch, Caesar habe in voller Absicht so hochmütig gehandelt. Seine öffentlichen Reaktionen auf die Ehrungen der vorangehenden Wochen als Indizien nehmend, sehen sie die In-

83 Bezieht man hier das weitere Publikum ein, kann der Versuch durchaus als erfolgreich bewertet werden, sofern das Bild des Opfers in den Augen der Zuschauer herabgesetzt wird. 
tentionalität auch beim Sitzenbleiben als gegeben an. Wie die Interaktion verläuft und ob es tatsächlich zu einer Gesichtsverletzung kommt, wird mithin nicht unerheblich durch die Wahrnehmung des Rezipienten und des Zuschauerkreises beeinflusst.

Im Falle des Sitzenbleibens hing der Erfolg des Kommunikationsaktes freilich nicht ausschließlich an der Intention des Akteurs und der Wahrnehmung durch das Publikum. Für Caesar selbst zeitigte der absichtliche Regelverstoß fatale Konsequenzen; er brachte das Fass zum Überlaufen und gab den entscheidenden Anstoß für die Ermordung des Dictators. Caesar hatte den Bogen überspannt und nicht bedacht, dass er selbst auf dem Gipfel der Macht der Bewertung durch die öffentliche Meinung ausgesetzt war und eine Gegenreaktion provozierte, mochte sie auch mit Verzögerung erfolgen. Zudem scheint er nicht ausreichend berücksichtigt zu haben, welche Beharrungskraft soziale Normen besitzen, dass nämlich auch die singuläre Stellung eines Dictators nicht davon enthebt, auf einige Konventionen Rücksicht zu nehmen, selbst wenn diese nicht mehr den realen Verhältnissen entsprechen. Das Publikum blieb keineswegs, wie er gehofft haben mochte, auf die passive Rolle des Zuschauers beschränkt, sondern indem es seinen Unmut zum Ausdruck brachte, ermöglichte es den Gegenschlag der Verschwörer, die einer breiteren $\mathrm{Zu}$ stimmung gewiss sein konnten. Wer allgemein akzeptierte Normen verletzt, so demonstriert die Episode, provoziert stets Missfallen und Kritik, da es Zeitgenossen gibt, welche den Regelverstoß als ungebührliches Ausscheren aus der Reihe bewerten. Gesellschaftliche Sanktionen, möglicherweise die physische Vernichtung, sorgen dann dafür, dass die Ordnung restituiert und das störende Individuum ausgegrenzt und unschädlich gemacht wird. Daraus ersehen wir schließlich, dass der kalkulierte Regelverstoß bei dem Akteur eine große kognitive Kompetenz voraussetzt. Gefordert wird von ihm nicht nur, die Rolle und die möglichen Reaktionen seiner unmittelbaren Mitspieler im voraus einzuschätzen, sondern ebenso die Haltung der Öffentlichkeit, also der zuschauenden Mitspieler. Er kann, wenn er sein Gesicht zu Ungunsten anderer konstituiert, nicht umhin, eine Kosten-Nutzen-Rechnung aufzumachen, also die Wirkung der Normentransgression abzuschätzen. ${ }^{84}$ Sueton ist dies nicht entgangen, insofern er in den einschlägigen Kapiteln wiederholt auf Caesars Ungeschick im Umgang mit der Öffentlichkeit anspielt. ${ }^{85}$ Augustus sollte sich als gelehriger Schüler erweisen, da er es verstand, im Umgang mit seinen Untertanen deren Gesicht zu wahren und den Eindruck zu erwecken, er respektiere die überkommene Ordnung. ${ }^{86}$ Sein Verhalten gegenüber Magistraten und Senatoren kann geradezu als Gegenentwurf zu Caesars Konfrontationskurs gelesen werden und als Lehrstück gelten, wie ein

84 Nur bei Plu. Caes. 6o,6 und App. BC 2,454 gelangt Caesar, wenn auch zu spät, zu der Einsicht, dass er mit dem Sitzenbleiben einen entscheidenden Fehler begangen, also die Situation falsch eingeschätzt hat.

85 Suet. Iul. 76,3: licentia spreto patrio more; 77: inpotentia, arrogantia; 79,I: multo arrogantius factum; vgl. Plu.

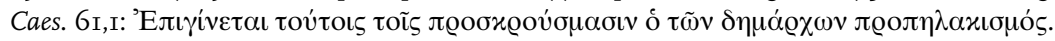

86 Einen scharfen Kontrast bildet vor allem die Szene des Jahres 22, in der Augustus die Dictatur angetragen wird. Statt wie Caesar durch seine Pose auszudrücken, dass diese Ehre selbstverständlich sei, fällt Augustus auf die Knie und entblößt seine Brust (Suet. Aug. 52). Augustus behandelt die Senatoren demonstrativ mit Respekt (ebd. 53,3; siehe ferner 56; D.C. 54,30,I). Flaig (2003) II6f. 
absoluter Herrscher durch Anpassung positiven Einfluss auf die öffentliche Meinung nehmen kann.

Das nötige Fingerspitzengefühl fehlte ebenso wie dem Dictator dem israelischen Außenministerium, das zum einen die Heftigkeit und das Gewicht der türkischen Reaktion unterschätzte und zum anderen von der Ablehnung in Teilen der israelischen Öffentlichkeit überrascht wurde. Symbolische Kommunikationsakte, die durch eine Normentransgression konstituiert werden, verlangen eine besondere Umsicht, wenn sie nicht nachteilige Folgen auslösen sollen. Insofern war Caesars Sitzenbleiben nicht nur ein geplanter Affront, sondern auch ein unbeabsichtigter ,Fehltritt', nämlich eine Beschädigung des eigenen Gesichts. Was der Dictator übersehen hatte, war, dass auch sein Gesicht nicht in seiner Hand lag, sondern von der Gesellschaft nur geliehen war.

\section{Literaturverzeichnis}

Aldrete (1999)

Gregory S. Aldrete, Gestures and Acclamations in Ancient Rome, Baltimore u. London.

Amici (199I)

Carla Maria Amici, Il foro di Cesare (Il linguaggio dell'architettura romana 2), 2 Bde., Florenz.

Bachmann-Medick (2006)

Doris Bachmann-Medick, Cultural Turns. Neuorientierungen in den Kulturwissenschaften, Reinbek.

\section{Bartsch (1994)}

Shadi Bartsch, Actors in the Audience. Theatricality and Doublespeak from Nero to Hadrian, Cambridge (MA) u. London.

\section{Boegehold (I999)}

Alan L. Boegehold, When a Gesture Was Expected. A Selection of Examples from Archaic and Classical Greek Literature, Princeton (NJ).

\section{Bremmer (1992)}

Jan Bremmer, „Walking, Standing, and Sitting in Ancient Greek Culture“, in: Jan Bremmer u. Herman Roodenburg (Hgg.), A Cultural History of Gesture, Ithaca (NY), I5-35.

\section{Bremmer u. Roodenburg (1992)}

Jan Bremmer u. Herman Roodenburg (Hgg.), A Cultural History of Gesture, Ithaca (NY).

Cairns (2005)

Douglas Cairns (Hg.), Body Language in the Greek and Roman Worlds, Swansea.

Corbeill (2004)

Anthony Corbeill, Nature Embodied. Gesture in Ancient Rome, Princeton (NJ) u. Oxford. 


\section{Davies (2005)}

Glenys Davies, „On Being Seated. Gender and Body Language in Hellenistic and Roman Art", in: Douglas Cairns (Hg.), Body Language in the Greek and Roman Worlds, Swansea, $215-238$.

\section{Dobesch (1988)}

Gerhard Dobesch, „Zu Caesars Sitzenbleiben vor dem Senat und zu der Quelle des Cassius Dio“, Tyche 3, 39-102. [wieder in: ders., Ausgewählte Schriften, hg. von Herbert Heftner u. Kurt Tomaschitz, Bd. I, Köln u.a. 200I, 275-36I]

\section{Dupont (2003)}

Florence Dupont, L'acteur-roi ou le théâtre dans la Rome antique, 2. Aufl., Paris.

Eich (2000)

Armin Eich, Politische Literatur in der römischen Gesellschaft. Studien zum Verhältnis von politischer und literarischer Öffentlichkeit in der späten Republik und frühen Kaiserzeit (Passauer historische Forschungen II), Köln u.a.

\section{Fischer-Lichte (2004)}

Erika Fischer-Lichte, Ästhetik des Performativen, Frankfurt/Main.

Flaig (2003)

Egon Flaig, Ritualisierte Politik. Zeichen, Gesten und Herrschaft im Alten Rom (Historische Semantik I), Göttingen.

\section{Fögen (2009)}

Thorsten Fögen (Hg.), Tears in the Graeco-Roman World, Berlin u. New York.

\section{Fuhrmann (1979)}

Manfred Fuhrmann, „Persona, ein römischer Rollenbegriff“, in: Odo Marquard u. Karlheinz Stierle (Hgg.), Identität (Poetik und Hermeneutik 8), München, 83-Io6.

\section{Gill (1988)}

Christopher Gill, „Personhood and Personality. The Four-personae Theory in Cicero, De officiis I“, Oxford Studies in Ancient Philosophy 6, I69-199.

\section{Goffman (1969)}

Erving Goffman, Wir alle spielen Theater. Die Selbstdarstellung im Alltag, München. [The Presentation of Self in Everyday Life, New York 1959]

\section{Goffman (1986)}

Erving Goffman, Interaktionsrituale. Über Verhalten in direkter Kommunikation, Frankfurt/ Main. [Interaction Ritual. Essays in Face-to-Face Behavior, New York I967]

\section{Graf (1992)}

Fritz Graf, „Gestures and Conventions. The Gestures of Roman Actors and Orators“, in: Jan Bremmer u. Herman Roodenburg (Hgg.), A Cultural History of Gesture, Ithaca (NY), $36-58$. 


\section{Grice (1979)}

Herbert Paul Grice, „Logik und Konversation“, in: Georg Meggle (Hg.), Handlung, Kommunikation, Bedeutung, Frankfurt/Main, 243-265 [„Logic and Conversation“, in: Peter Cole u. Jerry L. Morgan (Hgg.), Syntax and Semantics 3. Speech Acts, New York u.a. I975, 4I-58].

\section{Gros (1995)}

Pierre Gros, „Forum Iulium: Venus Genetrix, aedes“, in: Eva M. Steinby (Hg.), Lexicon Topographicum Urbis Romae, Bd. 2, Rom, $306 \mathrm{f}$.

\section{Jehne (1987)}

Martin Jehne, Der Staat des Dictators Caesar (Passauer historische Forschungen 3), Köln u. Wien.

\section{Jehne (2000)}

Martin Jehne, „Jovialität und Freiheit. Zur Institutionalität der Beziehungen zwischen Ober- und Unterschichten in der römischen Republik“, in: Bernhard Linke u. Michael Stemmler (Hgg.), Mos maiorum. Untersuchungen zu den Formen der Identitätsstiftung und Stabilisierung in der römischen Republik (Historia ES I4I), Stuttgart, 207-235.

Kolb (1977)

Frank Kolb, „Zur Statussymbolik im antiken Rom“, Chiron 7, 239-259.

\section{Kunkel u. Wittmann (1995)}

Wolfgang Kunkel u. Roland Wittmann, Staatsordnung und Staatspraxis der Römischen Republik. Zweiter Abschnitt: Die Magistratur (Handbuch der Altertumswissenschaft Io.3.2.2), München.

\section{Lintott (2009)}

Andrew Lintott, „The Assassination“, in: Miriam Griffin (Hg.), A Companion to Julius Caesar, Malden (MA), 72-82.

\section{Maier-Eichhorn (1989)}

Ursula Maier-Eichhorn, Die Gestikulation in Quintilians Rhetorik (Europäische Hochschulschriften 15,4I), Frankfurt/Main u.a.

\section{Mommsen (1887/8)}

Theodor Mommsen, Römisches Staatsrecht, 3 Bde., 3. bzw. I. Aufl., Leipzig.

von Moos (200I)

Peter von Moos, „Vorwort“, in: ders. (Hg.), Der Fehltritt. Vergehen und Versehen in der Vormoderne (Norm und Struktur I5), Köln u.a., XI-XXIV.

\section{Morselli (1995)}

Chiara Morselli, „Forum Iulium“, in: Eva M. Steinby (Hg.), Lexicon Topographicum Urbis Romae, Bd. 2, Rom, 299-306.

Procopé (I99I)

John Procopé, „Höflichkeit“, in: RAC I5, 930-986. 


\section{Ronke (1987)}

Jutta Ronke, Magistratische Repräsentation im römischen Relief. Studien zu standes- und statusbezeichnenden Szenen (BAR International Series 370), Oxford.

\section{Schäfer (1989)}

Thomas Schäfer, Imperii insignia. Sella curulis und fasces. Zur Repräsentation römischer Magistrate (Mitteilungen des DAI, römische Abteilung, Ergänzungsheft 29), Mainz.

\section{Sittl (I890)}

Carl Sittl, Die Gebärden der Griechen und Römer, Leipzig [ND Hildesheim I970].

\section{Stamper (2005)}

John W. Stamper, The Architecture of Roman Temples. The Republic to the Middle Empire, Cambridge.

\section{Stenger (2010)}

Jan Stenger, „,Im Theater erkennt man das Wesen eines Volkes.' Dion von Prusa und die symbolische Kommunikation in den Städten des Reiches“, in: Norbert Kramer u. Christiane Reitz (Hgg.), Tradition und Erneuerung. Mediale Strategien in der Zeit der Flavier (Beiträge zur Altertumskunde 285), Berlin u. New York, 397-427.

\section{Strocka (2005)}

Volker Michael Strocka, „Das Fassaden-Motiv des Venus Genetrix-Tempels in Rom. Bedeutung und Nachwirkung“, in: Stephan T. A. M. Mols u. Eric M. Moormann (Hgg.), Omni pede stare. Saggi architettonici e circumvesuviani in memoriam Jos de Waele, Neapel, I53-I67.

\section{Turner (1989)}

Victor Turner, Vom Ritual zum Theater. Der Ernst des menschlichen Spiels, Frankfurt/Main. [From Ritual to Theatre. The Human Seriousness of Play, New York I982]

\section{Ulrich (1993)}

Roger B. Ulrich, „Julius Ceasar and the Creation of the Forum Iulium“, AJA 97, 49-80.

\section{Ulrich (1994)}

Roger B. Ulrich, The Roman Orator and the Sacred Stage. The Roman Templum Rostratum (Collection Latomus 222), Brüssel.

\section{Weinstock (I97I)}

Stefan Weinstock, Divus Julius, Oxford.

\section{Wülfing (1995)}

Peter Wülfing, „Antike und moderne Redegestik. Eine frühe Theorie der Körpersprache bei Quintilian“, in: Gerhard Binder u. Konrad Ehlich (Hgg.), Kommunikation durch Zeichen und Wort. Stätten und Formen der Kommunikation im Altertum IV (Bochumer Altertumswiss. Colloquium 23), Trier, 7I-90. 